\title{
Extraordinary Altruists Exhibit Enhanced Self-Other Overlap in Neural Responses to Distress
}

Psychological Science 2018, Vol. 29(10) 1631-1641 (C) The Author(s) 2018 Article reuse guidelines: sagepub.com/journals-permissions DOI: $10.1177 / 0956797618779590$ www.psychologicalscience.org/PS @SAGE

\author{
Kristin M. Brethel-Haurwitz ${ }^{1}$ (D), Elise M. Cardinale ${ }^{2}$, \\ Kruti M. Vekaria ${ }^{2}$ (D, Emily L. Robertson ${ }^{3}$, Brian Walitt $^{4}$, \\ John W. VanMeter ${ }^{5}$, and Abigail A. Marsh' \\ ${ }^{1}$ Department of Psychology, University of Pennsylvania; ${ }^{2}$ Department of Psychology, Georgetown University; \\ ${ }^{3}$ Department of Psychology, Louisiana State University; ${ }^{4}$ National Institute of Nursing Research, National Institutes \\ of Health, Bethesda, Maryland; and ${ }^{5}$ Department of Neurology, Georgetown University Medical Center
}

\begin{abstract}
Shared neural representations during experienced and observed distress are hypothesized to reflect empathic neural simulation, which may support altruism. But the correspondence between real-world altruism and shared neural representations has not been directly tested, and empathy's role in promoting altruism toward strangers has been questioned. Here, we show that individuals who have performed costly altruism (donating a kidney to a stranger; $n=25)$ exhibit greater self-other overlap than matched control participants $(n=27)$ in neural representations of pain and threat (fearful anticipation) in anterior insula (AI) during an empathic-pain paradigm. Altruists exhibited greater self-other correspondence in pain-related activation in left AI, highlighting that group-level overlap was supported by individual-level associations between empathic pain and firsthand pain. Altruists exhibited enhanced functional coupling of left AI with left midinsula during empathic pain and threat. Results show that heightened neural instantiations of empathy correspond to real-world altruism and highlight limitations of self-report.
\end{abstract}

\section{Keywords}

altruism, empathy, neural simulation, nondirected living kidney donation

Received 9/20/17; Revision accepted 5/7/18

Does empathy promote altruism? Empathy is the suite of processes that enables an individual to perceive and understand others' distress, promoting the motivation to act on their behalf (de Waal, 2008). Empathy for pain entails recruiting neural processes that are equivalent to those recruited during the firsthand experience of pain (Rütgen et al., 2015), and such self-other mapping has been theorized to promote nonreciprocal altruism: voluntary, costly actions aimed at improving another person's welfare (Ashar, Andrews-Hanna, Dimidjian, \& Wager, 2017; Batson, 2010; de Waal, 2008; Preston \& de Waal, 2002). But linking empathy unambiguously to altruism has proven challenging. Some researchers argue that empathy may actually preclude altruism by biasing respondents away from individuals who are distant or dissimilar to them and that empathy must be overridden by cognitively mediated compassion (Bloom,
2017). The difficulty of objectively measuring and operationalizing both empathy and altruism impedes clear conclusions. Empathic self-other mapping cannot be directly reported or observed through behavior, and genuinely costly altruism is impractical or unethical to reproduce in a laboratory. The aim of the present study was to test putative links between objectively measured empathy and costly real-world altruism by evaluating whether individuals who have voluntarily engaged in a stringently defined form of altruism (donation of a kidney to a stranger) exhibit spontaneous enhancements

\section{Corresponding Author:}

Kristin M. Brethel-Haurwitz, University of Pennsylvania, Department of Psychology, 433 S. University Ave., Kable Lab-Goddard 5,

Philadelphia, PA 19104

E-mail: kbreth@sas.upenn.edu 
in self-other neural mapping of experienced and observed distress, including pain and threat (fearful anticipation).

The anterior insula (AI) is a neural region strongly implicated in empathy. Self-other mapping of pain can be observed in AI, which is active during both experienced and observed pain (Bernhardt \& Singer, 2012; Fan, Duncan, de Greck, \& Northoff, 2011; Lamm, Decety, \& Singer, 2011; Rütgen et al., 2015; Singer et al., 2004; Zaki, Wager, Singer, Keysers, \& Gazzola, 2016), particularly in response to close others or in-group members (Beckes, Coan, \& Hasselmo, 2012; Hein, Silani, Preuschoff, Batson, \& Singer, 2010), and has been implicated in affective empathy and nonreciprocal altruism more generally (Fan et al., 2011; Kanske, Bockler, Trautwein, \& Singer, 2015; Patil et al., 2017; Tusche, Bockler, Kanske, Trautwein, \& Singer, 2016). Only altruism for strangers can be unambiguously described as nonreciprocal; if empathy promotes nonreciprocal altruism, enhanced self-other neural mapping in AI and associated regions while viewing an anonymous stranger's pain may be observed in individuals who have engaged in costly altruism for strangers.

To test this hypothesis, we recruited a rare sample of individuals who had donated a kidney to a stranger and compared them with demographically matched control participants. Both groups underwent a pressurepain paradigm and also watched as a stranger underwent an identical pain paradigm in real time. Altruistic kidney donation is a voluntary, extraordinarily costly form of altruism performed to benefit strangers, thereby satisfying stringent definitions of altruism (Batson, 2010; Clavien \& Chapuisat, 2013; de Waal, 2008). Evaluating altruistic kidney donors circumvents ethical and practical considerations that preclude reproducing genuinely costly altruism in the laboratory and minimizes social desirability and norm-adherence motives that contaminate laboratory-elicited altruism (Eisenberg \& Fabes, 1990). The study of this special population of extraordinary altruists therefore provides valuable insight into the basis of altruism, much as the study of other special populations has provided important insights into mechanisms underlying, for example, memory (LePort et al., 2012) and face processing (Russell, Duchaine, \& Nakayama, 2009).

We hypothesized that enhanced group-level selfother mapping in AI and other affective pain regions would be observed in altruists relative to controls. Moreover, these group-level differences would be supported by increased self-other correspondence at the individual level during two types of pain-related distress (defined as negative affect elicited in response to or anticipation of an aversive stimulus). The two types of distress examined here were threat (fearful anticipation following a cue that probabilistically predicted pressure pain in the ensuing trial) and pressure pain. In other words, not only would altruists show more overlap of activation during experienced and empathic pain, and during experienced and empathic threat, but also experience-based activation would be associated with empathic activation in altruists at the individual level. Functional connectivity of AI during empathic pain and threat was also examined given AI's role as a hub in an affective salience network (Bernhardt \& Singer, 2012; Menon \& Uddin, 2010) that may support empathically motivated altruism.

\section{Method}

\section{Participants}

Fifty-seven participants, who included 29 altruistic kidney donors and 28 matched controls, took part in this study for monetary payment. Sample sizes were determined using fMRIPower (SPM Toolkit; Mumford \& Nichols, 2008), specialized software used to determine power estimates for functional MRI (fMRI) data, and pilot data from our previous study of altruistic donors (Marsh et al., 2014). One altruist was unable to complete the task as a result of scanner malfunction. Additionally, 3 altruists and 1 control participant were excluded from analyses for movement exceeding $0.5 \mathrm{~mm}$ for at least $15 \%$ of the total number of task repetition times (TRs). This resulted in a final sample of 25 altruistic kidney donors and 27 matched controls between 21 and 55 years old (see Table 1 for participant characteristics).

Altruistic kidney donors were recruited using mailings and electronic advertisements through local and national transplant organizations. The sample of altruists was limited by the extreme rarity of this behavior (1,819 such donations in the United States through 2016 according to the Organ Procurement and Transplantation Network, 2017). Because altruists were recruited from across North America, most altruists resided more than a 2 -hr drive from the university and were provided with airfare and up to two nights lodging. All altruists had donated a kidney to a stranger unknown to them personally at the time they decided to donate. Nineteen altruists were nondirected donors for whom the recipient was anonymous at the time of donation. The remaining 6 directed their donations to a specific individual who was known to them at the time of donation but whose need for a kidney they had learned about through, for example, a flier or an Internet posting. All donations were verified through independent sources, including transplant center records or media reports.

Using data obtained from the Organ Procurement and Transplantation Network, which is administered by the United Network of Organ Sharing under contract 
Table 1. Participant Characteristics

\begin{tabular}{lcccc}
\hline Characteristic & Altruists $(n=25)$ & Controls $(n=27)$ & $p$ & Effect size $^{\mathrm{a}}$ \\
\hline Male & $n=9(36.0 \%)$ & $n=13(48.2 \%)$ & .376 & 0.12 \\
White & $n=23(92.0 \%)$ & $n=22(81.5 \%)$ & .267 & 0.15 \\
Right-handed & $n=24(96.0 \%)$ & $n=25(92.6 \%)$ & .599 & 0.07 \\
Household income $\geq \$ 60,000$ & $n=19(76.0 \%)$ & $n=24(92.3 \%)$ & .109 & 0.22 \\
Education $\geq 4$-year degree & $n=17(68.0 \%)$ & $n=24(88.9 \%)$ & .065 & 0.26 \\
Age (years) & $M=41.92[38.04,45.80]$ & $M=38.89[35.38,41.40]$ & .229 & $0.34[-0.21,0.88]$ \\
IQ & $M=108.28[103.40,113.16]$ & $M=111.22[106.96,115.48]$ & .375 & $0.25[-0.30,0.79]$ \\
\hline
\end{tabular}

Note: One control subject did not report household income. For age and IQ, 95\% confidence intervals are given in brackets.

${ }^{a}$ Effect sizes for gender, race, handedness, income, and education are $\varphi s$; effect sizes for age and IQ are $d s$.

with the U.S. Department of Health and Human Services, we confirmed that the altruists recruited for this study were representative of the national population of altruistic donors in terms of sex and race (exact ages are not available for the national sample). Healthy volunteers were recruited from the local community using fliers, online advertisements, and electronic participant databases including ResearchMatch. The present study was conducted 3 years after our prior neuroimaging studies (Brethel-Haurwitz et al., 2017; Marsh et al., 2014) and included partially overlapping subject samples, with 12 altruists and 6 controls returning for this study.

Exclusion criteria for all participants included current use of psychotropic medication, history of head injury or neurological illness, IQ less than 80 (as assessed using the Kaufman Brief Intelligence Test-Second Edition; Kaufman \& Kaufman, 2004), and pregnancy or other contraindications to safe MRI scanning, including metal fragments or implants. Thorough screening of psychopathology was also conducted to ensure group matching on potentially relevant psychological variables. Participants were excluded if they scored above clinical cutoffs for Global Severity, Positive Symptom Distress, or Positive Symptom Total on the Symptom Checklist-90 (Derogatis \& Unger, 2010) Somatization, Obsessive-Compulsive, Depression, Anxiety, Paranoid Ideation, or Psychoticism subscales. If totals reflected elevated scores on the Interpersonal Sensitivity or Hostility subscales, participants were not excluded. Additionally, given the nature of the task, participants were excluded if they reported a pain disorder or hearing difficulties.

Controls were excluded if they reported having ever volunteered to donate an organ to any individual (not including consenting to become a deceased organ donor) or if they expressed interest in potentially doing so. Specifically, all controls were asked during the initial screening if they would be interested in receiving additional information from the Washington Regional
Transplant Center about becoming a living organ donor, and 27 potential controls who answered yes to this question were excluded solely for this reason. All study procedures were approved by the Georgetown University Institutional Review Board, and all participants provided written informed consent before testing.

\section{Procedure}

All interested volunteers initially completed a 90-min online survey assessing exclusion and inclusion criteria, demographic variables, and several self-report questionnaires including the Interpersonal Reactivity Index (IRI; Davis, 1983). The IRI is a 28 -item questionnaire that measures both total self-reported empathy and four subscales: Empathic Concern, Perspective Taking, Fantasy Scale, and Personal Distress. Eligible volunteers were then screened by telephone to confirm eligibility. Researchers coordinated altruists' travel to and lodging at Georgetown University to enable on-site neuroimaging and cognitive testing. On-site testing included the Empathy for Pain Scale (Giummarra et al., 2015), which is a self-report questionnaire measuring empathy specific to pain through 12 emotion constructs across four contexts in which pain may be observed, resulting in three subscales: Empathic Concern, Affective Distress, and Vicarious Pain. To ensure groups were matched, we asked eligible controls to complete a laboratory screening that included assessments of IQ, income, education, psychological history, medication use, and MRI compatibility before MRI scanning. After confirmation of eligibility, controls completed neurocognitive tasks and MRI scanning in a final visit.

During the calibration procedure and the fMRI task (see Neuroimaging Task), participants received painful pressure stimulation to their right thumbnail. Pneumatic pressure was administered remotely by an MRI-safe device connected to a laptop computer with software programmed to consistently administer a selected pressure level at intervals and durations matching the task 
a

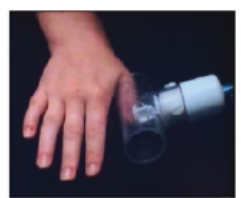

Study Partner

b

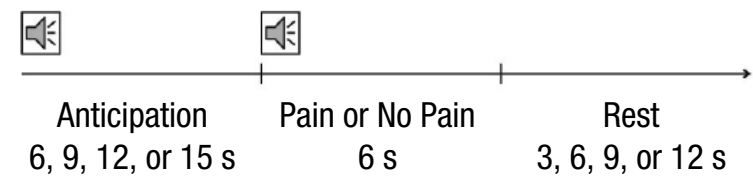

Fig. 1. The empathic-pain task. Participants viewed continuous live video feeds (a) of the hand of the study partner (confederate) and then their own hand, in separate runs. On each trial (b), a variable anticipation period was followed by the administration or omission of painful pressure stimulation to the right thumbnail. An audio cue at the beginning of the anticipation period indicated whether the trial was safe or had the potential for pain. A second audio cue indicated whether or not painful pressure was administered.

structure. When pressure was administered, a circular plastic plunger with a 2 -cm diameter rubber surface made contact with the right thumbnail of the participant or confederate, whose thumb was positioned in a clear plastic tube to keep it in place throughout the task (see Fig. 1a). Pressure of a rectangular waveform was administered for $6 \mathrm{~s}$.

Two live video feeds allowed each participant to observe both the hand of the confederate and his or her own hand during the task (Fig. 1a). A video camera positioned next to the confederate in the MRI console room captured video of the confederate's hand. Each participant was briefly introduced to the confederate in this setup, so that it was apparent to the participant that the video feed would in fact be live. An MRI-safe video camera positioned adjacent to the scanner was adjusted for each participant to capture an equivalent view of the participant's own right hand receiving painful stimulation. The video feed was switched between these two cameras depending on the run of the task.

\section{Neuroimaging task}

Participants completed three runs of the pain task, each lasting $12 \mathrm{~min}$ and $18 \mathrm{~s}$, during fMRI scanning. In the first two runs, participants observed a "study partner," and in the third run they experienced the task themselves (Fig. 1a). The study partner was a female confederate whom participants were briefly introduced to immediately prior to the scan. Two confederates participated over the course of the study, balanced between the two groups. The second run of the task began with a prompt to encourage increased empathizing with the study partner; results for this task are not reported here to limit the current analyses to group differences in baseline responses to the observation of pain in another person relative to the firsthand experience of pain. The effect of the empathy prompt will be reported in a separate article.

Each run consisted of 30 trials, with each trial made up of a variable anticipation period, the administration or omission of painful pressure stimulation, and a variable rest period (Fig. 1b). Within each block, half of the trials were safe trials, in which participants knew that there would be no thumb pressure; the other half of the trials were threat trials, in which participants knew there was a potential for thumb pressure. Pressure was omitted on one third of these potentially painful trials to keep the administration of pain probabilistic rather than deterministic, which is important to elicit fearful anticipation (Sylvers, Lilienfeld, \& LaPrairie, 2011); this design resulted in 10 pain trials and 20 nopain trials within each block.

Throughout each trial, participants viewed continuous live video feeds of either the confederate's hand (in the first and second runs) or their own hand (in the third run). Audio cues via MRI-safe headphones indicated the trial structure: A first sound indicated at the start of each trial whether it was a safe trial or had the potential for painful pressure, and a second sound after the anticipation period indicated whether or not pressure was currently being administered. Participants were trained in the meaning of the various audio cues prior to the scan, reminded of the cue meanings once in the scanner, and quizzed on their comprehension prior to the first run. Participants' ability to hear the audio cues and differentiate them from scanner noise was confirmed during a localizer scan prior to the first run.

Immediately prior to scanning, all participants were guided through a calibration procedure to determine the level of pressure to be administered during the scan; this was done so subjective pain perceptions were equivalent across participants. All participants were calibrated to receive pain that was subjectively "slightly intense," which corresponded to a rating of 13.5 on the 21-point Gracely Box Scale (Gracely \& Kwilosz, 1988), which was selected to be moderately painful but not harmful.

Following explanation of the Gracely Box Scale and the calibration procedure, increasing pressure levels were administered, beginning with 5 psi and increasing by increments of 5 psi until participants gave a rating of 13.5. Ascending pressure was continued until a rating 
Table 2. Analysis of Ratings of Intensity of Subjective Pain, Pain Unpleasantness, and Fear

\begin{tabular}{lcccc}
\hline $\begin{array}{l}\text { Variable and } \\
\text { condition }\end{array}$ & Altruists $(M)$ & Controls $(M)$ & $p$ & $d$ \\
\hline $\begin{array}{l}\text { Pain } \\
\text { Self }\end{array}$ & $4.48[4.00,4.96]$ & $4.30[3.81,4.79]$ & .603 & $0.15[-0.40,0.69]$ \\
$\quad$ Other & $4.08[3.66,4.50]$ & $3.93[3.62,4.24]$ & .564 & $0.16[-0.38,0.71]$ \\
Unpleasantness & & & & \\
$\quad$ Self pain & $3.60[3.06,4.14]$ & $3.85[3.32,4.38]$ & .527 & $0.18[-0.37,0.73]$ \\
$\quad$ Self no pain & $1.16[1.01,1.31]$ & $1.27[1.01,1.53]$ & .476 & $0.20[-0.35,0.75]$ \\
$\quad$ Other pain & $3.84[3.34,4.34]$ & $3.81[3.20,4.42]$ & .936 & $0.02[-0.53,0.57]$ \\
$\quad$ Other no pain & $1.44[1.03,1.85]$ & $1.12[0.99,1.25]$ & .137 & $0.42[-0.13,0.98]$ \\
Fear & & & & \\
$\quad$ Self threat & $3.36[2.76,3.96]$ & $3.65[3.20,4.10]$ & .442 & $0.22[-0.33,0.77]$ \\
$\quad$ Self no threat & $1.16[1.01,1.31]$ & $1.15[1.01,1.29]$ & .953 & $0.02[-0.53,0.57]$ \\
$\quad$ Other threat & $3.48[2.94,4.02]$ & $3.92[3.47,4.37]$ & .222 & $0.35[-0.21,0.90]$ \\
$\quad$ Other no threat & $1.12[0.99,1.25]$ & $1.23[1.03,1.43]$ & .367 & $0.25[-0.30,0.80]$ \\
\hline
\end{tabular}

Note: For means and effect sizes, 95\% confidence intervals are given in brackets. This pattern of results was the same when we controlled for objective pain level.

higher than 13.5 was given, then descending pressure in increments of 5 psi was administered until participants gave a rating lower than 13.5. If different, the lower of the two pressure levels rated 13.5 was selected as the "slightly intense" pressure level. The pressure selected during the calibration procedure was checked in the scanner immediately prior to the run in which participants received pressure themselves. Several participants in each group ( 8 altruists and 10 controls), $\chi^{2}(1)=0.15, p=.703, \varphi=0.05$, rated the pressure selected during calibration more highly than 13.5 once in the scanner, resulting in a recalibration procedure in which descending pressure in increments of 5 psi was tested until participants gave a rating of 13.5. This recalibrated lower pressure was then the pressure administered to the participant during the scan. Because altruists selected significantly higher objective pressure for their subjectively calibrated "slightly intense," $t(50)=$ $3.97, p<.001, d=1.10,95 \%$ confidence interval (CI) for the effect size $=[0.51,1.68]$ (Fig. S1b in the Supplemental Material available online), the final pressure level was included as a covariate in analyses of experienced pain and threat. The confederate always received 15 psi of pressure, which was selected to be clearly visible over the live video feed and within the range of slightly intense pain for both groups.

Subjective ratings of pain experience were collected during and after scanning. Following each run, participants were asked to rate pain intensity on a scale from 1 (no pain) to 7 (extreme pain). For Run 1, this was their perception of the study partner's pain level, whereas for Run 3 this was their rating of their own pain experience. After the scan, participants filled out a questionnaire in which they rated how fearful or anxious their experience during the anticipation portion of the task was and how unpleasant their experience during the pain portion of the task was, both on a scale from 1 (not at all) to 7 (extremely). They also answered the same questions regarding the experience of their study partner.

Importantly, despite significantly different objective pressure stimulation levels selected as "slightly intense" by altruists and controls (Fig. S1b), subjective experiences of threat and pain were equivalent between the two groups. There were no group differences in subjective ratings of threat or pain during or after the scan for the participants' firsthand experience or their perception of the study partner's experience (all $p s>.05$; Table 2). Across all participants, cues indicating that pain may follow were rated as more fear and anxiety inducing than safety cues, $t(50)=12.55, p<.001, d=$ 2.38 , supporting the interpretation that this anticipation epoch induced fearful anticipation. Further, thumbnail stimulation was rated as more unpleasant than the omission of thumbnail stimulation, $t(50)=13.02, p<$ $.001, d=2.41$. Threat of pain, $t(50)=14.04, p<.001$, $d=2.64$, and the experience of thumbnail stimulation, $t(50)=12.02, p<.001, d=2.22$, were also rated negatively for the study partner, relative to the safety trials and omission of stimulation.

Participants also rated their perception of connectedness to others on the Inclusion of Other in the Self Scale (Aron, Aron, \& Smollan, 1992). Connectedness was rated by selecting one of seven diagrams of overlapping circles representing perceived connectedness to the other individual, from 1 (separate and distant circles, 
a

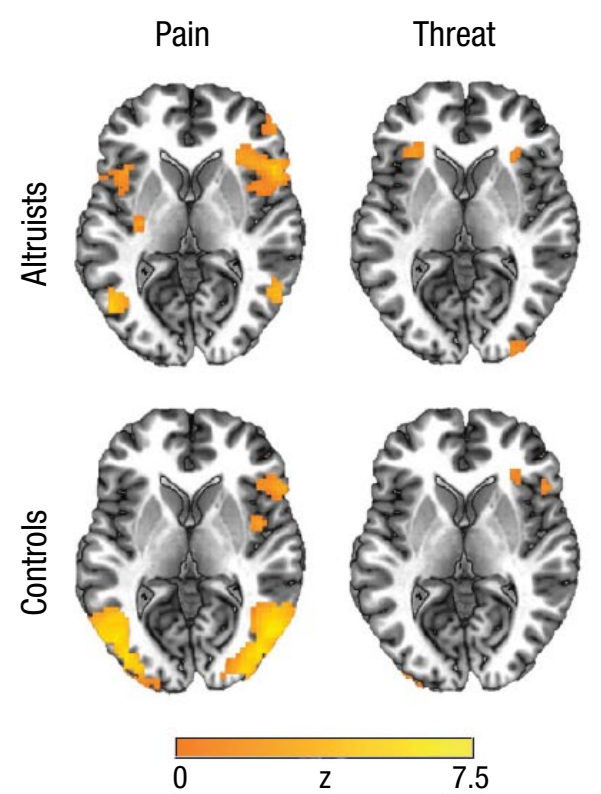

b

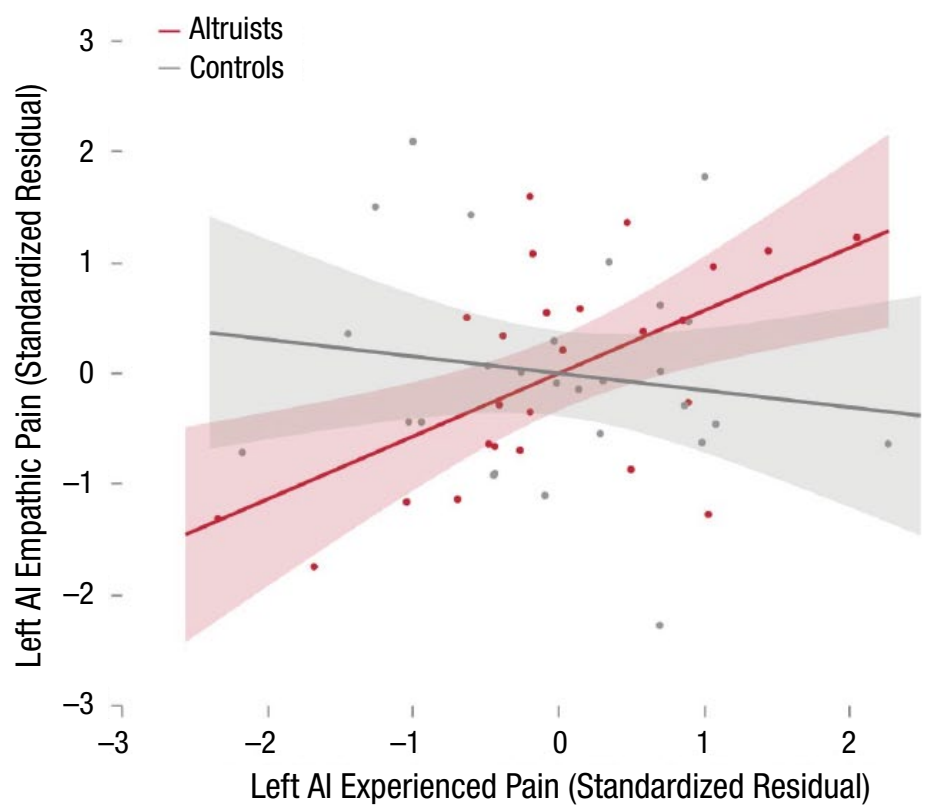

3

Fig. 2. Empathic pain and threat conjunction and covariation results. Whole-brain statistical maps (a) show the conjunction of neural activation for experienced and empathic pain and the conjunction of neural activation for experienced and empathic threat, separately for each group $(z=0)$. A whole-brain clusterwise corrected threshold $p<.05$ at uncorrected voxelwise threshold of $p=.001$ was applied to the statistical maps. The scatterplot (b) shows the relationship between activations in the left AI region of interest for experienced pain and empathic pain. Standardized residuals and lines of best fit are plotted, controlling for objective pain level. Shading represents $95 \%$ confidence intervals.

representing the lowest degree of connectedness) to 7 (completely overlapping circles, representing the highest degree of connectedness). Relationships rated included immediate family, extended family, friend, acquaintance, stranger, and study partner from the empathic-pain task. Data from 1 control participant were missing for the experience questionnaire, and 1 altruist did not complete the Inclusion of Other in the Self Scale.

All reported statistical tests were two-tailed. Correlations between behavioral variables and neural activation in the AI regions of interest (ROIs) are reported in Table S1 in the Supplemental Material. Only significant correlations surviving correction for multiple comparisons via the Benjamini-Hochberg method of controlling the false discovery rate $(\mathrm{Q}<.05)$ are reported here. For additional details on neuroimaging acquisition and analysis, see the Supplemental Material.

\section{Results}

First, whole-brain conjunction analyses were conducted to compare activation for experienced and empathic pain. Individuals who have engaged in costly altruism for a stranger spontaneously exhibited greater self-other conjunction in bilateral AI while observing an anonymous stranger's pain; specifically, increased activation in bilateral AI emerged during both experienced and observed pain (corrected $p<.05$ ). Both altruists and controls exhibited overlap in bilateral inferior parietal cortex and right AI, but only altruists exhibited overlap in left AI (Fig. 2a). In altruists only, conjunctions were also observed in putamen, thalamus, dorsomedial prefrontal cortex, and middle cingulate cortex (Table S2 in the Supplemental Material). A similar pattern was observed for distinct epochs of experienced and empathic threat, during which conjunction in bilateral AI as well as dorsomedial prefrontal cortex was found in altruists, whereas conjunction in controls was limited to right $\mathrm{AI}$, right caudate, and left inferior parietal lobule (corrected $p<.05$; see Fig. $2 \mathrm{a}$ as well as Table S3 in the Supplemental Material). Reinforcing that these group differences reflect specifically empathic processes, differences in self-other mapping across groups emerged despite comparable activation for experienced pain and threat across groups (see Fig. S1 and Tables S4 and S5 in the Supplemental Material). Examining activation levels in AI ROIs for experienced and empathic pain and threat, we found no group differences, even when controlling for objective pain level (all ps $>.05, d s<0.48$; Fig. S2 in the Supplemental Material). There were also no significant differences within any condition in wholebrain group contrasts. 
Mean parameter estimates were extracted for ROIs in bilateral AI to examine individual-level correspondence in activation levels for experienced and empathic pain and threat. Partial correlations controlling for objective pain intensity confirmed group-level findings for experienced pain in left AI. In altruists, activation in left AI during firsthand pain experience was associated with activation in the identical region when observing an anonymous stranger experiencing pain, $r(22)=.57,95 \% \mathrm{CI}=[.22, .79], p=.004$, whereas no similar relationship was observed in controls, $r(24)=$ $-.15,95 \% \mathrm{CI}=[-.51, .25], p=.456$ (Fig. 2b); these correlations differed significantly in magnitude $(z=2.64$, $p=.008$ ). This effect was phenomenologically specific, in that left AI activation during pain experience was not associated with left AI activation for empathic threat in altruists, $r(22)=-.23,95 \% \mathrm{CI}=[-.58, .19], p=.286$. Neither altruists, $r(22)=.19,95 \% \mathrm{CI}=[-.23, .55], p=$ .380 , nor controls, $r(24)=.22,95 \% \mathrm{CI}=[-.18, .56], p=$ .291, exhibited self-other covariation in right AI. Parallel partial correlations during threat were also examined. In this analysis, no self-other covariation was observed in altruists' left AI, $r(22)=.29,95 \% \mathrm{CI}=[-.13$, $.62], p=.168$, or right AI, $r(22)=.01,95 \% \mathrm{CI}=[-.40$, .41], $p=.965$. The same was true for controls in both the left AI, $r(24)=-.02,95 \% \mathrm{CI}=[-.40, .37], p=.932$, and right $\mathrm{AI}, r(24)=.30,95 \% \mathrm{CI}=[-.10, .62], p=.137$. Pain self-other conjunction and covariation results held when repeated in two control analyses: when excluding directed altruistic donors and when including education and income levels as covariates. However, threat selfother conjunction results are less robust. ${ }^{1}$

Given the insula's theorized role as a hub of a neural network critical in processing the salience of internal and external stimuli (Bernhardt \& Singer, 2012; Craig, 2009; Lamm \& Singer, 2010; Menon \& Uddin, 2010) and the observed differences between altruists and controls in self-other mapping in left AI for the neural processing of pain and threat, group differences in functional connectivity between a left AI seed and a priori ROIs were examined. During both empathic pain and empathic threat, altruists exhibited greater functional connectivity than controls between left AI and left midinsula (corrected $p<.05$; Table S6 in the Supplemental Material). During empathic threat, at a less stringent voxelwise cluster-forming significance threshold of .005 , altruists also exhibited greater functional connectivity between left AI and left amygdala. Repeating the two sets of control analyses, in which functional connectivity results were examined in a reduced sample of altruists and with education and income covariates, revealed mixed results. ${ }^{2}$ Altruists' greater functional connectivity between left AI and left midinsula during empathic pain persisted in the reduced sample but not following the inclusion of demographic covariates. In contrast, altruists' greater functional connectivity between the left AI and left midinsula and left amygdala during empathic threat persisted with demographic covariates but not in the reduced sample.

Altruists' enhanced self-other neural conjunction for a stranger's distress may reflect increased subjective valuation of the welfare of strangers (Vekaria, BrethelHaurwitz, Cardinale, Stoycos, \& Marsh, 2017). Consistent with this, altruists reported higher inclusion of other in the self (a measure that corresponds to feelings of connectedness) with the study partner, $t(48)=2.16$, $p=.036, d=0.61,95 \% \mathrm{CI}=[0.04,1.18]$, and, at a trend level, strangers in general, $t(48)=2.00, p=.051, d=$ $0.57,95 \% \mathrm{CI}=[0.00,1.13]$. Altruists' and controls' reported connectedness with objectively closer relationships did not differ (all $p s>.05, d s<0.28$; Fig. $\mathrm{S} 3$ in the Supplemental Material). Inclusion of rated connectedness with the specific anonymous stranger experiencing pain in this study as a covariate in whole-brain self-other conjunction analysis of the threat condition eliminated self-other conjunction in right $\mathrm{AI}$ in altruists. By comparison, if rated connectedness with immediate family was instead added as a covariate in this conjunction analysis, bilateral AI self-other conjunction for threat in altruists was maintained. Feelings of connectedness with a stranger in distress may therefore partially mediate altruists' empathic responsiveness, particularly during the experience of threat.

In light of ongoing debates about the value of empathy in promoting concern and altruism (Bloom, 2017), we investigated the relationship between self-reported empathy and outcome variables. Despite altruists and controls exhibiting objectively measurable differences in empathic responding in this paradigm, they did not differ in self-reported total or subscale general empathy, including empathic concern (all $p s>.05, d s<0.44$ ). Altruists and controls also did not differ on any subscale of self-reported empathy for pain (all $p \mathrm{~s}>.05, d \mathrm{~s}<$ 0.48). In altruists only, both general empathic concern, $r(22)=.60,95 \% \mathrm{CI}=[.26, .81], p=.002$, and empathic concern specifically for pain, $r(22)=.58,95 \% \mathrm{CI}=[.23$, $.80], p=.003$, were associated with ratings of connectedness with strangers. Neither measure of self-reported empathic concern was associated with neural measures of empathy in either group, as indexed by activation in AI ROIs during empathic pain or threat, when controlling for multiple comparisons (Table S1). Thus-unlike measures of empathy at the neural level-neither selfreport measure corresponded to real-world altruism, reinforcing the need for caution when interpreting selfreport measures of empathy (Eisenberg \& Fabes, 1990). 


\section{Discussion}

The present findings directly link objectively measured empathy and altruism. Engagement in costly, nonreciprocal altruism for a stranger corresponded to spontaneous recruitment of shared neural representations while observing an anonymous stranger experiencing pain or threat. When altruists observed a stranger, activation in AI was more closely associated with activation during firsthand experience, particularly for pain. According to dominant models of empathy, shared representations of other individuals' distress, including pain and fear, are key drivers of altruistic helping (Batson, 2010). Extensive evidence highlights that empathic pain reflects activation in affective components of the neural pain matrix that are also active during personal experiences of somatic pain, including bilateral AI (e.g., Corradi-Dell'Acqua, Hofstetter, \& Vuilleumier, 2011; Lamm et al., 2011). That both experienced and empathic pain can be eliminated by placebo analgesia and reinstated using the opioid antagonist naltrexone supports the idea that both experienced pain and empathic pain rely on common neurochemical circuits (Rütgen et al., 2015). The anterior portion of the insula in particular is thought to be involved in representing subjective unpleasantness (Craig, Chen, Bandy, \& Reiman, 2000) and in integrating somatic and interoceptive information from middle and posterior insula (Craig, 2009). This ideally situates AI for processing both firsthand feelings and body states and similar states observed in others (Lamm \& Singer, 2010). Further, AI is hypothesized to be critical for learning and predicting emotional states in social settings (Singer, Critchley, \& Preuschoff, 2009) as a hub of a proposed salience network (Bernhardt \& Singer, 2012; Menon \& Uddin, 2010).

This study benefited from close matching of the firsthand experience of two distressing states-pain and threat-and the identical experiences observed in a stranger, allowing for a specific direct test of self-other mapping. During experienced and empathic conditions, participants viewed nearly identical live video feeds of their own and a confederate's right hand during painful pressure stimulation and anticipation of painful stimulation, within the same paradigm. Other studies have relied on secondary cues (e.g., Corradi-Dell'Acqua, Tusche, Vuilleumier, \& Singer, 2016) to indicate whether pain was occurring or on photos of painful situations distinct from the participant's experience (e.g., Krishnan et al., 2016; Lamm, Meltzoff, \& Decety, 2010; Lamm, Nusbaum, Meltzoff, \& Decety, 2007). While the current study aimed to match subjective experiences across groups, future investigations of empathic simulation in highly altruistic individuals could explore the effects of experienced and observed pain experiences of varying intensities, which could help to determine whether the patterns observed here are specific to pain, which has been the focus of recent debate (Corradi-Dell'Acqua et al., 2016; Krishnan et al., 2016; Zaki et al., 2016). Functional activation findings for empathic pain were robust to control analyses. Less robust findings when examining empathic threat and in analyses of functional connectivity possibly reflect the reduced statistical power of the control analyses but highlight the need for further study. It will be particularly important to assess the generalizability of neural simulation across distress-related emotions and how the insula functions within a neural network to encode empathic distress.

Altruistic kidney donors were selected as a sample of extraordinary altruists meeting the most stringent definitions of human altruism. This allowed us to examine how altruism unconfounded by social desirability or norm adherence biases may be related to neural instantiations of empathy, and generalizability to individuals who engage in more normative and less costly prosocial behaviors may be limited (Brethel-Haurwitz, Stoycos, Cardinale, Huebner, \& Marsh, 2016). Whether enhanced empathic neural simulation would also be observed in other highly altruistic individuals (e.g., heroic rescuers) or the extent to which empathic neural simulation can be predicted by less extreme prosocial behaviors will be an important area for future research.

The present findings are bolstered by the use of multiple approaches to examining self-other neural mapping, including conjunction, covariation, and connectivity analyses of functional activation. While both empathic pain and threat elicited bilateral self-other overlap in AI in altruists, individual-level covariation was specific to left AI during empathic pain. Further evidence for the specific role AI may play during empathic threat and pain was highlighted by functional connectivity analyses, in which functional connections between this region and other areas of the insula, and perhaps the amygdala, may support altruists' enhanced empathic response to distress in strangers. This suggest that AI functions as part of an affective salience network (Bernhardt \& Singer, 2012; Menon \& Uddin, 2010) that may support altruists' enhanced responsiveness to other people's distress (Marsh et al., 2014). That altruists, relative to controls, exhibit enhanced self-other mapping for strangers' distress-ordinarily observed only in response to close others-supports the hypothesis that empathic biases against distant or dissimilar others can be overcome and that, ultimately, empathy can support the provision of costly altruism.

\section{Action Editor}

Ralph Adolphs served as action editor for this article. 


\section{Author Contributions}

A. A. Marsh developed the study concept. A. A. Marsh, K. M. Brethel-Haurwitz, E. M. Cardinale, B. Walitt, and J. W. VanMeter designed the research. K. M. Brethel-Haurwitz, E. M. Cardinale, K. M. Vekaria, E. L. Robertson, J. W. VanMeter, and A. A. Marsh performed the research. K. M. Brethel-Haurwitz analyzed the data. K. M. Brethel-Haurwitz and A. A. Marsh drafted the manuscript. All the authors approved the final manuscript for submission.

\section{ORCID iDs}

Kristin M. Brethel-Haurwitz (D) https://orcid.org/0000-00030429-4598

Kruti M. Vekaria (D) https://orcid.org/0000-0003-3981-4637

\section{Acknowledgments}

We thank Alaina Pearce, Sarah Furlong, Abbey Hammell, and the Center for Functional and Molecular Imaging for assistance with data collection. We also thank the participants who contributed their time and energy to this work.

\section{Declaration of Conflicting Interests}

The author(s) declared that there were no conflicts of interest with respect to the authorship or the publication of this article.

\section{Funding}

This project was supported by John Templeton Foundation Grant 47861 to A. A. Marsh and National Institutes of Health National Center for Advancing Translational Sciences Grant 1KL2RR031974-01 to J. W. VanMeter.

\section{Supplemental Material}

Additional supporting information can be found at http:// journals.sagepub.com/doi/suppl/10.1177/0956797618779590

\section{Open Practices}

Data and neuroimaging analysis scripts are available through the Open Science Framework (https://osf.io/jyqa6/). The design and analysis plans were not preregistered.

\section{Notes}

1. The functional activation analyses and covariation analyses were repeated with the exclusion of 6 altruistic kidney donors who had donated to a specific stranger. In this reduced sample, altruists had significant bilateral self-other conjunction in AI for pain and a significant covariation between self and other pain activation in left $\mathrm{AI}, r(16)=.49,95 \% \mathrm{CI}=[.03, .78], p=$ .038. However, in this reduced sample, altruists had significant self-other conjunction only in left AI (not in right AI) for threat. Functional activation analyses and covariation analyses were also repeated with education level and household income as binary covariates of no interest, given trends toward significant group differences in these demographic variables. In this analysis controlling for education and income, altruists still had significant bilateral self-other conjunction in AI for pain and a significant covariation between self and other pain activation in left AI, $r(20)=.59,95 \% \mathrm{CI}=[.22, .81], p=.004$. In controls, there was still only significant self-other conjunction for pain in right $\mathrm{AI}$ and no pain self-other covariation in left AI, $r(21)=-.16$, $95 \% \mathrm{CI}=[-.54, .27], p=.477$. However, with these demographic covariates, altruists no longer had self-other threat conjunction in either AI, whereas controls had a small but significant conjunction in right AI (2 voxels).

2 . The functional connectivity analyses were also repeated with the reduced altruist sample and the two demographic covariates (education and income). With the reduced sample of altruists, there was still a significant group difference in which altruists had greater functional connectivity during empathic pain between left AI and left midinsula, relative to controls. However, in this reduced sample, there was no group difference in functional connectivity with left AI during empathic threat, even at a less stringent voxelwise significance threshold of .005. With demographic covariates, there were no group differences in functional connectivity with left AI for empathic pain, except for a significant cluster in left midinsula at the less stringent voxelwise threshold of .005 . For empathic threat, altruists had significantly greater functional connectivity between left AI and left midinsula and between left AI and left amygdala, relative to controls, with education and income as covariates. At a less stringent voxelwise threshold of .005 , there was also significantly greater functional connectivity with right amygdala for altruists during empathic threat, controlling for demographic covariates.

\section{References}

Aron, A., Aron, E. N., \& Smollan, D. (1992). Inclusion of Other in the Self Scale and the structure of interpersonal closeness. Journal of Personality and Social Psychology, 63, 596-612.

Ashar, Y. K., Andrews-Hanna, J. R., Dimidjian, S., \& Wager, T. (2017). Empathic care and distress: Predictive brain markers and dissociable brain systems. Neuron, 94, 1263-1273. doi:10.1016/j.neuron.2017.05.014

Batson, C. D. (2010). The naked emperor: Seeking a more plausible genetic basis for psychological altruism. Economics \& Philosophy, 26, 149-164. doi:10.1017/ S0266267110000179

Beckes, L., Coan, J. A., \& Hasselmo, K. (2012). Familiarity promotes the blurring of self and other in the neural representation of threat. Social Cognitive and Affective Neuroscience, 8, 670-677. doi:10.1093/scan/nss046

Bernhardt, B. C., \& Singer, T. (2012). The neural basis of empathy. Annual Review of Neuroscience, 35, 1-23. doi:10.1146/annurev-neuro-062111-150536

Bloom, P. (2017). Empathy and its discontents. Trends in Cognitive Sciences, 21, 24-31. doi:10.1016/j.tics.2016.11.004

Brethel-Haurwitz, K. M., O'Connell, K., Cardinale, E. M., Stoianova, M., Stoycos, S. A., Lozier, L. M., . . . Marsh, A. A. (2017). Amygdala-midbrain connectivity indicates a role for the mammalian parental care system in human altruism. Proceedings of the Royal Society B: Biological 
Sciences, 284(1865), Article 20171731. doi:10.1098/ rspb. 2017.1731

Brethel-Haurwitz, K. M., Stoycos, S. A., Cardinale, E. M., Huebner, B., \& Marsh, A. A. (2016). Is costly punishment altruistic? Exploring rejection of unfair offers in the ultimatum game in real-world altruists. Scientific Reports, 6, Article 18974. doi:10.1038/srep18974

Clavien, C., \& Chapuisat, M. (2013). Altruism across disciplines: One word, multiple meanings. Biology \& Philosophy, 28, 125-140. doi:10.1007/s10539-012-9317-3

Corradi-Dell'Acqua, C., Hofstetter, C., \& Vuilleumier, P. (2011). Felt and seen pain evoke the same local patterns of cortical activity in insular and cingulate cortex. The Journal of Neuroscience, 31, 17996-18006. doi:10.1523/ JNEUROSCI.2686-11.2011

Corradi-Dell'Acqua, C., Tusche, A., Vuilleumier, P., \& Singer, T. (2016). Cross-modal representations of first-hand and vicarious pain, disgust and fairness in insular and cingulate cortex. Nature Communications, 7, Article 10904. doi:10.1038/ncomms10904

Craig, A. D. (2009). How do you feel - now? The anterior insula and human awareness. Nature Reviews Neuroscience, 10, 59-70. doi:10.1038/nrn2555

Craig, A. D., Chen, K., Bandy, D., \& Reiman, E. M. (2000). Thermosensory activation of insular cortex. Nature Neuroscience, 3, 184-190. doi:10.1038/72131

Davis, M. H. (1983). Measuring individual differences in empathy: Evidence for a multidimensional approach. Journal of Personality and Social Psychology, 44, 113-126. doi:10.1037/0022-3514.44.1.113

Derogatis, L. R., \& Unger, R. (2010). Symptom Checklist90-Revised. In I. B. Weiner \& W. E. Craighead (Eds.), The Corsini encyclopedia of psychology (4th ed., pp. 1743-1744). Hoboken, NJ: John Wiley \& Sons. doi:10.1002/9780470479216.corpsy0970

de Waal, F. B. (2008). Putting the altruism back into altruism: The evolution of empathy. Annual Review of Psychology, 59, 279-300. doi:10.1146/annurev.psych.59.103006.093625

Eisenberg, N., \& Fabes, R. A. (1990). Empathy: Conceptualization, measurement, and relation to prosocial behavior. Motivation and Emotion, 14, 131-149.

Fan, Y., Duncan, N. W., de Greck, M., \& Northoff, G. (2011). Is there a core neural network in empathy? An fMRI based quantitative meta-analysis. Neuroscience \& Biobehavioral Reviews, 35, 903-911. doi:10.1016/j.neubiorev.2010.10.009

Giummarra, M. J., Fitzgibbon, B. M., Georgiou-Karistianis, N., Beukelman, M., Verdejo-Garcia, A., Blumberg, Z., ... Gibson, S. J. (2015). Affective, sensory and empathic sharing of another's pain: The Empathy for Pain Scale. European Journal of Pain, 19, 807-816. doi:10.1002/ejp.607

Gracely, R. H., \& Kwilosz, D. M. (1988). The descriptor differential scale: Applying psychophysical principles to clinical pain assessment. Pain, 35, 279-288.

Hein, G., Silani, G., Preuschoff, K., Batson, C. D., \& Singer, T. (2010). Neural responses to ingroup and outgroup members' suffering predict individual differences in costly helping. Neuron, 68, 149-160. doi:10.1016/j.neuron .2010 .09 .003
Kanske, P., Bockler, A., Trautwein, F. M., \& Singer, T. (2015). Dissecting the social brain: Introducing the EmpaToM to reveal distinct neural networks and brain-behavior relations for empathy and theory of mind. NeuroImage, 122, 6-19. doi:10.1016/j.neuroimage.2015.07.082

Kaufman, A. S., \& Kaufman, N. L. (2004). Kaufman Brief Intelligence Test-Second Edition (KBIT-2). Circle Pines, $\mathrm{MN}$ : American Guidance Service.

Krishnan, A., Woo, C. W., Chang, L. J., Ruzic, L., Gu, X., LópezSolà, M., . . . Wager, T. D. (2016). Somatic and vicarious pain are represented by dissociable multivariate brain patterns. elife, 5, Article e15166. doi:10.7554/eLife.15166

Lamm, C., Decety, J., \& Singer, T. (2011). Meta-analytic evidence for common and distinct neural networks associated with directly experienced pain and empathy for pain. NeuroImage, 54, 2492-2502. doi:10.1016/j.neuroimage .2010.10.014

Lamm, C., Meltzoff, A. N., \& Decety, J. (2010). How do we empathize with someone who is not like us? A functional magnetic resonance imaging study. Journal of Cognitive Neuroscience, 22, 362-376. doi:10.1162/jocn.2009.21186

Lamm, C., Nusbaum, H. C., Meltzoff, A. N., \& Decety, J. (2007). What are you feeling? Using functional magnetic resonance imaging to assess the modulation of sensory and affective responses during empathy for pain. PLOS ONE, 2(12), Article e1292. doi:10.1371/journal.pone.0001292

Lamm, C., \& Singer, T. (2010). The role of anterior insular cortex in social emotions. Brain Structure \& Function, 214, 579-591. doi:10.1007/s00429-010-0251-3

LePort, A. K., Mattfeld, A. T., Dickinson-Anson, H., Fallon, J. H., Stark, C. E., Kruggel, F., . . McGaugh, J. L. (2012). Behavioral and neuroanatomical investigation of highly superior autobiographical memory (HSAM). Neurobiology of Learning and Memory, 98, 78-92. doi:10.1016/j.nlm .2012 .05 .002

Marsh, A. A., Stoycos, S. A., Brethel-Haurwitz, K. M., Robinson, P., VanMeter, J. W., \& Cardinale, E. M. (2014). Neural and cognitive characteristics of extraordinary altruists. Proceedings of the National Academy of Sciences, USA, 111, 15036-15041. doi:10.1073/pnas.1408440111

Menon, V., \& Uddin, L. Q. (2010). Saliency, switching, attention and control: A network model of insula function. Brain Structure E Function, 214, 655-667. doi:10.1007/ s00429-010-0262-0

Mumford, J. A., \& Nichols, T. E. (2008). Power calculation for group fMRI studies accounting for arbitrary design and temporal autocorrelation. NeuroImage, 39, 261-268. doi:10.1016/j.neuroimage.2007.07.061

Organ Procurement and Transplantation Network. (2017). National data [Living donor transplants in the U.S. by state]. Retrieved from http://optn.transplant.hrsa.gov/data/

Patil, I., Zanon, M., Novembre, G., Zangrando, N., Chittaro, L., \& Silani, G. (2017). Neuroanatomical basis of concernbased altruism in virtual environment. Neuropsychologia. Advance online publication. doi:10.1016/j.neuropsy chologia.2017.02.015

Preston, S. D., \& de Waal, F. B. (2002). Empathy: Its ultimate and proximate bases. Behavioral E Brain Sciences, 25, 1-20. 
Russell, R., Duchaine, B., \& Nakayama, K. (2009). Superrecognizers: People with extraordinary face recognition ability. Psychonomic Bulletin \& Review, 16, 252-257. doi:10.3758/PBR.16.2.252

Rütgen, M., Seidel, E. M., Silani, G., Riećanský, I., Hummer, A., Windischberger, C., . . . Lamm, C. (2015). Placebo analgesia and its opioidergic regulation suggest that empathy for pain is grounded in self pain. Proceedings of the National Academy of Sciences, USA, 112, E5638-E5646. doi:10.1073/pnas.1511269112

Singer, T., Critchley, H. D., \& Preuschoff, K. (2009). A common role of insula in feelings, empathy and uncertainty. Trends in Cognitive Sciences, 13, 334-340. doi:10.1016/j .tics.2009.05.001

Singer, T., Seymour, B., O’Doherty, J., Kaube, H., Dolan, R. J., \& Frith, C. D. (2004). Empathy for pain involves the affective but not sensory components of pain. Science, 303, 1157-1162. doi:10.1126/science. 1093535
Sylvers, P., Lilienfeld, S. O., \& LaPrairie, J. L. (2011). Differences between trait fear and trait anxiety: Implications for psychopathology. Clinical Psychology Review, 31, 122-137. doi:10.1016/j.cpr.2010.08.004

Tusche, A., Bockler, A., Kanske, P., Trautwein, F.-M., \& Singer, T. (2016). Decoding the charitable brain: Empathy, perspective taking, and attention shifts differentially predict altruistic giving. The Journal of Neuroscience, 36, 4719-4732. doi:10.1523/JNEUROSCI.3392-15.2016

Vekaria, K. M., Brethel-Haurwitz, K. M., Cardinale, E. M., Stoycos, S. A., \& Marsh, A. A. (2017). Social discounting and distance perceptions in costly altruism. Nature Human Behaviour, 1, Article 0100. doi:10.1038/s41562-017-0100

Zaki, J., Wager, T. D., Singer, T., Keysers, C., \& Gazzola, V. (2016). The anatomy of suffering: Understanding the relationship between nociceptive and empathic pain. Trends in Cognitive Sciences, 20, 249-259. doi:10.1016/j .tics.2016.02.003 